\title{
Editorials
}

\section{Models of paramedic involvement in general practice}

\section{INTRODUCTION}

There is a continued increase in demand for both planned and same-day primary care. The reality that the number of qualified full-time equivalent GPs in England fell once more to under 29000 at the end of $2018,{ }^{1}$ means that new models of working are necessary if general practice is to withstand these pressures. In addition to committing to recruit and train more than 3000 additional GPs over the next few years, the NHS England GP Forward View proposes greater development of the multidisciplinary, integrated primary care workforce, capitalising on the value that allied healthcare professionals can bring support to front line service delivery. ${ }^{2}$ The recent announcement of funding for 20000 additional allied professionals and clinical support staff over the next 5 years further founds a trajectory that more patient care should be delivered by non-GPs. ${ }^{3}$ The GP Forward View specifically highlights the skills of paramedics, and suggests that general practice should look to make greater use of this professional group.? While acknowledging that scope of practice may vary, particular examples of perceived benefit include the management of minor illness, undertaking of home visits, and the provision of same day 'urgent' primary care.

There are already signs that these recommendations are being acted on. The General Practice Workforce dataset indicates that there were 513 paramedics working in practices in England as of December 2018 , a $71 \%$ increase on the previous year, outnumbering physician associates by more than three to one. ${ }^{1}$ There are also a variety of community admission-avoidance and "early visiting' services establishing, with some early work underway to try and understand what might drive success in these schemes. ${ }^{4}$ Despite the clear policy direction, very little is known about the safety, clinical-, or cost-effectiveness, of paramedics working in primary care. Models currently in use include paramedics seeing same-day and urgent problems only, undertaking chronic disease clinics, performing telephone triage, completing home visits, or a hybrid of one of more of these. There are also a variety of employment arrangements and variable degrees of association with other community services. Few models, if any, have been subject to rigorous, academic exploration. Just as the numbers of GPs are

\section{"The unselected nature of cases across the entire age and demographic spectrum is typical of a paramedic's sphere of practice, which is an attractive prospect for primary care}

squeezed, there is not an inexorable supply of paramedics. It is important to understand the true impact of a potential 'brain drain' on other areas of the health infrastructure, such as front-line ambulance services, before whole heartedly endorsing this professional shift.

\section{SUPPORT AT THE COALFACE: THE THEORIES}

Theories regarding how paramedics may support primary care centre around three main ideas:

- paramedics seeing routine patients will release GP time to manage other patients, particularly the more complex patients where continuity matters most;

- paramedics create additional capacity to see/visit urgent problems in a timely manner throughout the day, perhaps affording greater opportunity to access other community or ambulatory pathways; and,

- paramedics may improve patient satisfaction and possibly clinical outcomes if they are able to see patients quickly and spend more time with them than GPs can.

The premise that modern day paramedics are also trained as 'generalists' from the outset of their careers, arguably makes primary care a suitable and relevant domain in which to practice. The unselected nature of cases across the entire age and demographic spectrum is typical of a paramedic's sphere of practice, which is an attractive prospect for primary care. There are specialised skills required in a general practice setting that are less likely to be part of a paramedic's core capabilities. These include the routine management of multimorbidity and chronic disease, a shift towards preventative care, and a mastery of more nebulous concepts as 'continuity' and 'the therapeutic consultation; ; argued by some to be the cornerstones of the craft of general practice.
Others have suggested that the sheer crisis in primary care capacity at present negates these more nuanced issues, such that the existence of a professional group with an applicable set of core competencies should be wholeheartedly embraced for whatever support it can offer. As with other practitioner groups, paramedics will bring in additional skills and knowledge particularly in acute care - that may be less developed in the general practice setting. These workforce and skill-mix issues have been well discussed. ${ }^{5}$

\section{PARAMEDIC PRESCRIBING: DEFINING 'ADVANCED PRACTICE'}

The phenomenon of paramedics working in primary care will imminently step up a further gear, just as the first cohorts of fully-qualified independent paramedic prescribers started to put pen to prescription over recent months.

Prescribing by non-medical professionals is of course nothing new; legislation permitting nurses to prescribe was first drawn up in 1992, and further amended by the Health and Social Care Act 2001 to enable other health professionals such as pharmacists to lawfully independently prescribe. The recent legislation enabling paramedics to prescribe followed an extensive period of public and professional consultation. ${ }^{6}$ In order to independently prescribe, paramedics must be recorded as such on the Health and Care Professions Council (HCPC) by completing an approved programme of training.

There is an expectation that paramedics will be working at an 'advanced level of practice' before embarking on prescribing. Historically, terminology relating to 'advanced' practice has been inconsistently used, and has led to much confusion about specifically what different skills and level of autonomy a 'specialist' or 'advanced' paramedic might be expected to possess. Titles such as primary 


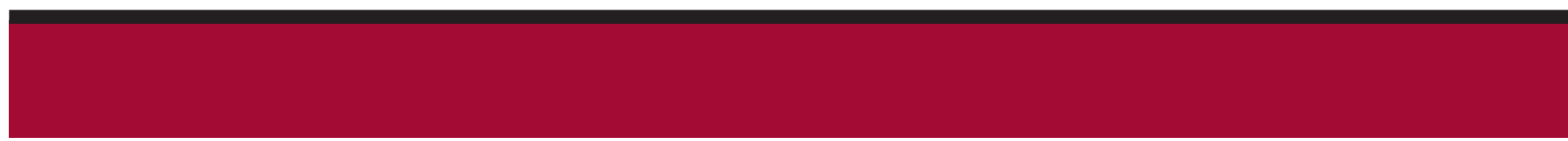

\section{"For primary care, the challenge will be in embedding these practitioners into the system in the most appropriate manner for a given context"}

\section{ADDRESS FOR CORRESPONDENCE}

\section{Matthew Booker}

Centre for Academic Primary Care, University of Bristol, Bristol BS8 2PS, UK.

\section{Email: matthew.bookeraBristol.ac.uk}

care paramedic practitioner' are also commonly used in job descriptions and policy literature. Important collaborative work has been led by bodies such as the College of Paramedics to standardise postregistration career framework descriptors, but roles such as 'advanced paramedic' are not always fully understood by patients or colleagues in primary care. Health Education England suggests advanced practice is ... characterised by a high degree of autonomy and complex decision making ... underpinned by a master'slevel award or equivalent ..., ${ }^{7}$ and has also produced a core capabilities framework for specialist paramedics working in primary care. $^{8}$ Before undertaking prescribing qualifications, paramedics are required to have a minimum of 3 years post-registration experience, and be able to demonstrate that they are working in a setting where there is an identified need to prescribe, with appropriate governance arrangements and support from employers.

The 'need' is likely to be uncontested if the paramedic is working in a general practice or community primary care setting, and GP surgeries that employ other nonmedical prescribers are likely to have all the necessary governance arrangements already in place. Some caution has been levelled against the interpretation of the 'advanced' practice benchmark, as some institutions appear to take a broader interpretation of what constitutes advanced practice when offering places on prescribing courses. While there are those who voice caution about potential increases in unnecessary prescriptions (such as antimicrobials), there is no compelling evidence that this is any more of a problem for one professional prescribing group than another.

\section{ALL PARAMEDICS AND GP SURGERIES ARE NOT EQUAL}

For primary care, the challenge will be in embedding these practitioners into the system in the most appropriate manner for a given context. Different models are likely to be required to take account of the different professional backgrounds, capabilities, experience, and skill sets. The types of patients seen, the clinical problems managed, and the relationships with other acute and community services, will almost certainly need to be tailored to specific local need. The impact of the transition of a skilled workforce from ambulance services to primary care services must also be evaluated, particularly at a time where policy advocates the treatment of more ambulance cases 'at scene'. It seems likely that there will be no "one size fits all' approach to how paramedics are best deployed to support general practice, with different practice sizes, workforce composition, and demographics, requiring a range of ways of working. This presents a significant challenge for robust evaluation of what is most cost- and resource-effective, or which model improves access and clinical outcomes.

It has been argued that, with paramedicine becoming an increasingly academic discipline in its own right, the evaluation of the emerging role of the prescribing 'advanced primary care paramedic' should be driven by the profession itself. ${ }^{10}$ Understanding the impacts of the ever more closely overlapping professional remits will require a collaborative and flexible research approach that can adapt to a constantly shifting landscape. While the face value arguments for paramedics in general practice may seem logical and clear, research is needed to understand if and why it works, how it can be sustainably scaled, and what the impacts are on other parts of the system. Research by our team and others is underway to address this.

\section{Matthew Booker,}

NIHR Clinical Lecturer in Primary Care, Centre for Academic Primary Care, University of Bristol, Bristol, UK

\section{Sarah Voss,}

Associate Professor in Emergency Care, Centre for Health and Clinical Research, University of the West of England, Bristol, UK.

\section{Provenance}

Commissioned; externally peer reviewed.

\section{Competing interests}

The authors have declared no competing interests.

DOI: https://doi.org/10.3399/bjgp19X705605

\section{REFERENCES}

1. NHS Digital. General practice workforce, England, December 2018 final: experimental statistics. 2019. https://digital.nhs.uk/dataand-information/publications/statistical/ general-and-personal-medical-services/final31-december-2018 laccessed 30 Aug 2019).

2. NHS England. General practice forward view. 2016. https://www.england.nhs.uk/ wp-content/uploads/2016/04/gpfv.pdf laccessed 30 Aug 2019).

3. NHS England. Investment and evolution: a five-year framework for GP contract reform to implement the NHS Long Term Plan. 2019. https://www.england.nhs.uk/wp-content/ uploads/2019/01/gp-contract-2019.pdf laccessed 30 Aug 2019).

4. Abrams R, Wong G, Mahtani KR, et al. Understanding the impact of delegated home visiting services accessed via general practice by community-dwelling patients: a realist review protocol. BMJ Open 2018; 8(11): e024876.

5. Nelson P, Martindale A, McBride A, et al. Skill-mix change and the general practice work-force challenge. Br J Gen Pract 2018; DOI: https://doi.org/10.3399/bjgp18X694469.

6. NHS England. Summary of the responses to the public consultation on proposals to introduce independent prescribing by paramedics across the United Kingdom. 2016 https://www.england.nhs.uk/wp-content/ uploads/2016/02/Paramedics-summaryconsult-responses.pdf laccessed 30 Aug 2019).

7. Health Education England. Multi-professional framework for advanced clinical practice in England. 2017. https://www.hee.nhs. uk/sites/default/files/documents/Multiprofessional\%20framework $\% 20$ for $\% 20$ advanced\%20clinical\%20practice $\% 20$ in $\% 20$ England.pdf (accessed 30 Aug 2019).

8. Health Education England. Paramedic specialist in primary and urgent care core capabilities framework. https://www.hee. nhs.uk/sites/default/files/documents/ Paramedic\%20Specialist\%20in\%20 Primary $\% 20$ and $\% 20$ Urgent $\% 20$ Care $\% 20$ Core\%20Capabilities\%20Framework.pdf laccessed 30 Aug 2019).

9. Limb M. Ambulances should treat more and transport fewer patients, NHS Improvement says. BMJ 2018; 362: k4106

10. Eaton G, Mahtani K, Catterall M. The evolving role of paramedics - a NICE problem to have? J Health Serv Res Policy 2018; 23(3): 193-195 